
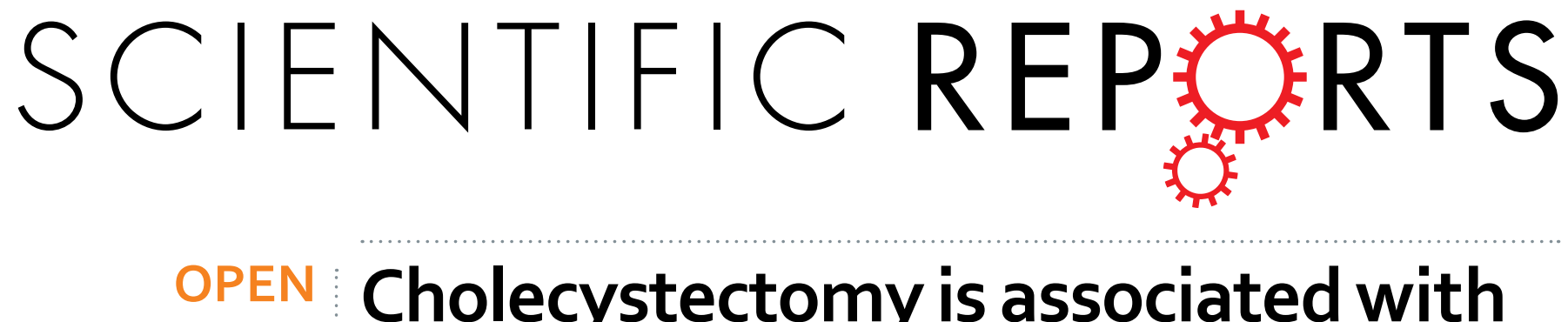

\title{
Cholecystectomy is associated with higher risk of early recurrence and poorer survival after curative
}

Received: 08 January 2016

Accepted: 31 May 2016

Published: 20 June 2016 resection for early stage hepatocellular carcinoma

Tao $\mathrm{Li}^{1,}{ }^{,}$, Shu-Kang Wang ${ }^{2,}{ }^{,}$, Xu-Ting Zhi ${ }^{1,}{ }^{*}$, Jian Zhou ${ }^{3}$, Zhao-Ru Dong ${ }^{1}$, Zong-Li Zhang ${ }^{1}$, Hui-Chuan Sun ${ }^{3}$, Qing-HaiYe ${ }^{3} \&$ Jia Fan ${ }^{3}$

Although cholecystectomy has been reported to be associated with increased risk of developing hepatocellular carcinoma (HCC), the association between cholecystectomy and prognosis of $\mathrm{HCC}$ patients underwent curative resection has never been examined. Through retrospective analysis of the data of 3933 patients underwent curative resection for HCC, we found that cholecystectomy was an independent prognostic factor for recurrence-free survival (RFS) of patients at early stage (BCLC stage $0 / A)(p=0.020$, HR: $1.29,95 \% \mathrm{Cl}: 1.04-1.59)$, and the 1-, 3-, 5-year RFS rates for patients at early stage were significantly worse in cholecystectomy group than in non-cholecystectomy group $(80.5 \%, 61.8 \%, 52.0 \%$ vs $88.2 \%$, $68.8 \%, 56.8 \%, p=0.033$ ). The early recurrence rate of cholecystectomy group was significantly higher than that of non-cholecystectomy group for patients at early stage ( $59 / 47$ vs $236 / 333, p=0.007)$, but not for patients at advanced stage (BCLC stage $C)(p=0.194)$. Multivariate analyses showed that cholecystectomy was an independent risk factor for early recurrence $(p=0.005, \mathrm{HR}: 1.52,95 \% \mathrm{Cl}$ : $1.13-2.03)$ of early stage HCC, but not for late recurrence $(p=0.959)$. In conclusion, cholecystectomy is an independent predictor for early recurrence and is associated with poorer RFS of early stage HCC. Removal of normal gallbladder during $\mathrm{HCC}$ resection may be avoided for early stage patients.

For more than a century cholecystectomy has been a method of choice in surgical management of gallbladder diseases, and the declining incidence of gallbladder cancer is considered to related to the increasing cholecystectomy rates ${ }^{1}$. Recently, much more epidemiological investigations and meta-analyses have addressed the relation between cholecystectomy and hepatocellular carcinoma $(\mathrm{HCC})^{2,3-5}$. Though a causal association between them remains to be established, the majority of these studies indicate that cholecystectomy is associated with an increased risk of developing HCC.

The reason for increased risk of developing HCC after cholecystectomy is sill unclear. One potential mechanism may involve chronic inflammation. Cholecystectomy can not only cause dilation of the CBD, elevated bile duct pressure, subsequent cholestasis and infection ${ }^{6-8}$, but also can induce systemic inflammation and cause increase of postoperative Interleukin (IL)-1, IL-6 and C-reactive protein (CRP) ${ }^{9-11}$. The link between chronic inflammation and HCC is well established ${ }^{12}$. Persistent inflammation will not only cause necrosis and regeneration of hepatocytes, thereby lead to DNA instability in the hepatocytes, and cause HCC to occur more frequently ${ }^{13-15}$, but also stimulate the release of cytokines, chemokines, vascular adhesion molecules and reactive oxygen species ${ }^{5,16-18}$, all of which play decisive roles in cancer development.

Previous studies of HCC have revealed various risk factors related to postoperative outcome $\mathrm{e}^{19-21}$. The severity of virally induced inflammation, which was well correlated with viral serostatus, has been established as an adverse risk factor for postoperative recurrence ${ }^{22-24}$, and antiviral therapy during the surveillance period is associated with

${ }^{1}$ Department of General Surgery, Qilu Hospital, Shandong University, Jinan, P.R. China. ${ }^{2}$ Department of Biostatistics, School of Public Health, Shandong University, Jinan, P.R. China. ${ }^{3}$ Liver Cancer Institute, Zhongshan Hospital, Fudan University, Shanghai, P.R. China. "These authors contributed equally to this work. Correspondence and requests for materials should be addressed to T.L. (email: litao7706@163.com) 


\begin{tabular}{|c|c|c|c|}
\hline Variable & $\begin{array}{c}\text { Non-cholecystectomy } \\
(\mathrm{n}=3294)\end{array}$ & $\begin{array}{l}\text { Cholecystectomy } \\
(n=639)\end{array}$ & p value \\
\hline BCLC stage (\%) & & & $<0.001$ \\
\hline $0 / \mathrm{A}$ & $2015(61.2)$ & $307(48.0)$ & \\
\hline B & $956(29.0)$ & $209(32.7)$ & \\
\hline $\mathrm{C}$ & $323(9.8)$ & $123(19.3)$ & \\
\hline Resection range (\%) & & & $<0.001$ \\
\hline Minor ( $\leq 2$ Couinaud segments) & $2288(69.5)$ & $356(55.7)$ & \\
\hline Major (>2 Couinaud segments) & $1006(30.5)$ & $283(44.3)$ & \\
\hline \multicolumn{4}{|l|}{ Postoperative complications (\%) } \\
\hline Bile leakage & $159(4.8)$ & $35(5.6)$ & 0.487 \\
\hline Hemorrhage & $19(0.6)$ & $3(0.5)$ & 0.739 \\
\hline Intra-abdominal abscess & $29(0.9)$ & $8(1.3)$ & 0.373 \\
\hline Pleural effusion & $201(6.1)$ & $47(7.4)$ & 0.233 \\
\hline Mean recurrence time(Median), mo & $17.9(12)$ & $13.2(9)$ & $<0.001$ \\
\hline Recurrence, early/late & $573 / 499$ & $165 / 86$ & $<0.001$ \\
\hline BCLC stage 0/A & $236 / 333$ & $59 / 47$ & 0.007 \\
\hline BCLC stage B & $229 / 141$ & $63 / 23$ & 0.048 \\
\hline BCLC stage $\mathrm{C}$ & $108 / 25$ & $43 / 16$ & 0.194 \\
\hline
\end{tabular}

Table 1. Tumor staging, operative variables and postoperative outcomes of HCC patients in cholecystectomy and non-cholecystectomy group.

reduced recurrence and improvement in overall survival in HBV-related $\mathrm{HCC}^{25,26}$. The decreased incidence of recurrence in patients who have used nonsteroidal anti-inflammatory drugs (NSAIDs), regardless of patients' viral hepatitis status, is supportive of a role for non-virus related inflammation in HCC recurrence ${ }^{27}$. Since an association is biologically plausible, the relation between cholecystectomy and outcome of HCC merits investigation. In this study, we retrospectively analyzed the data of 3933 consecutive HCC patients treated by curative resection, to investigate the impact of cholecystectomy on postoperative recurrence and survival of HCC patients.

\section{Results}

Patient distribution, survival and recurrence rates according to BCLC staging. A total of 3933 HCC patients received curative resection for HCC (The clinicopathologic characteristics of all 3933 HCC patients are listed in supplementary Table 1), among them, 639 patients also underwent cholecystectomy simultaneously. Of these 639 patients, 71 patients had gallbladder disease: acute cholecystitis in 1 patient; adenomyoma in 13 patients; gallstones in 40 patients; polyp in 8 patients; and HCC invasion or metastasis of the gallbladder in 9 patients. The overall prevalence of gallbladder invasion or metastasis from HCC was $0.2 \%$ (9/3933). Of the other 568 patients with normal gallbladder removed, 99 patients underwent right hemihepatectomy, 85 patients underwent left hemihepatectomy, 28 patients underwent central hepatectomy, 356 patients underwent local or segment resection and the gallbladder was removed because the tumor was adjacent to the gallbladder. All patients were followed up and the median follow-up time was 20 months (range, 1-168 months).

Further tumor staging according to the Barcelona Clinic Liver Cancer (BCLC) staging system revealed that the ratio of patients at early stage (BCLC stage 0/A) was significantly higher in no-cholecystectomy group than in cholecystectomy group $(61.2 \%$ vs $48.0 \%, \mathrm{p}<0.001$, Table 1$)$. Though the ratio of patients received major resection was significantly higher in cholecystectomy group than in no-cholecystectomy group (283/639 vs 1006/3294, $\mathrm{p}<0.001$, Table 1), there were no significant differences regarding postoperative complications including bile leakage $(\mathrm{p}=0.478)$, hemorrhage $(\mathrm{p}=0.739)$, intra-abdominal abscess $(\mathrm{p}=0.373)$ and pleural effusion $(\mathrm{p}=0.233)$.

The incidence of intrahepatic recurrence of cholecystectomy group was significantly higher than that of no-cholecystectomy group $(1072 / 3294$ vs $251 / 639, \mathrm{p}=0.001)$. The early recurrence rate of cholecystectomy group were also significantly higher than that of no-cholecystectomy group $(65.7 \%$ vs $53.5 \%, \mathrm{p}<0.001$, Table 1$)$. However, when patients were stratified according to BCLC stage, the difference was significant only for patients at early stage $(p=0.007$, Table 1$)$ or intermediate stage (BCLC stage $B)(p=0.048$, Table 1$)$, but not for patients at advanced stage (BCLC stage C) $(\mathrm{p}=0.194$, Table 1$)$.

Stratified analysis of overall survival (OS) according to BCLC staging system demonstrated no significant difference between the cholecystectomy group and no-cholecystectomy group at early, intermediate or advanced stage (Fig. 1A-C, p = 0.337, 0.397 and 0.449, respectively), and only for patients at early stage, the recurrence free survival (RFS) rates of cholecystectomy group were significantly worse than those of non-cholecystectomy group $(80.5 \%, 61.8 \%, 52.0 \%$ vs $88.2 \%, 68.8 \%, 56.8 \%$, Fig. $1 \mathrm{D}, \mathrm{p}=0.033)$. The RFS of patients at intermediate or advanced stage did not differ significantly between the cholecystectomy group and no-cholecystectomy group (Fig. 1E,F, $\mathrm{p}=0.226,0.853$, respectively).

Comparison of clinicopathologic characteristics between early stage HCC patients in no-cholecystectomy group and cholecystectomy group. The clinicopathologic characteristics of early stage HCC patients in no-cholecystectomy group and cholecystectomy group were compared in Table 2. Despite age and tumor size, there were no significant differences between these two groups regarding serum level 

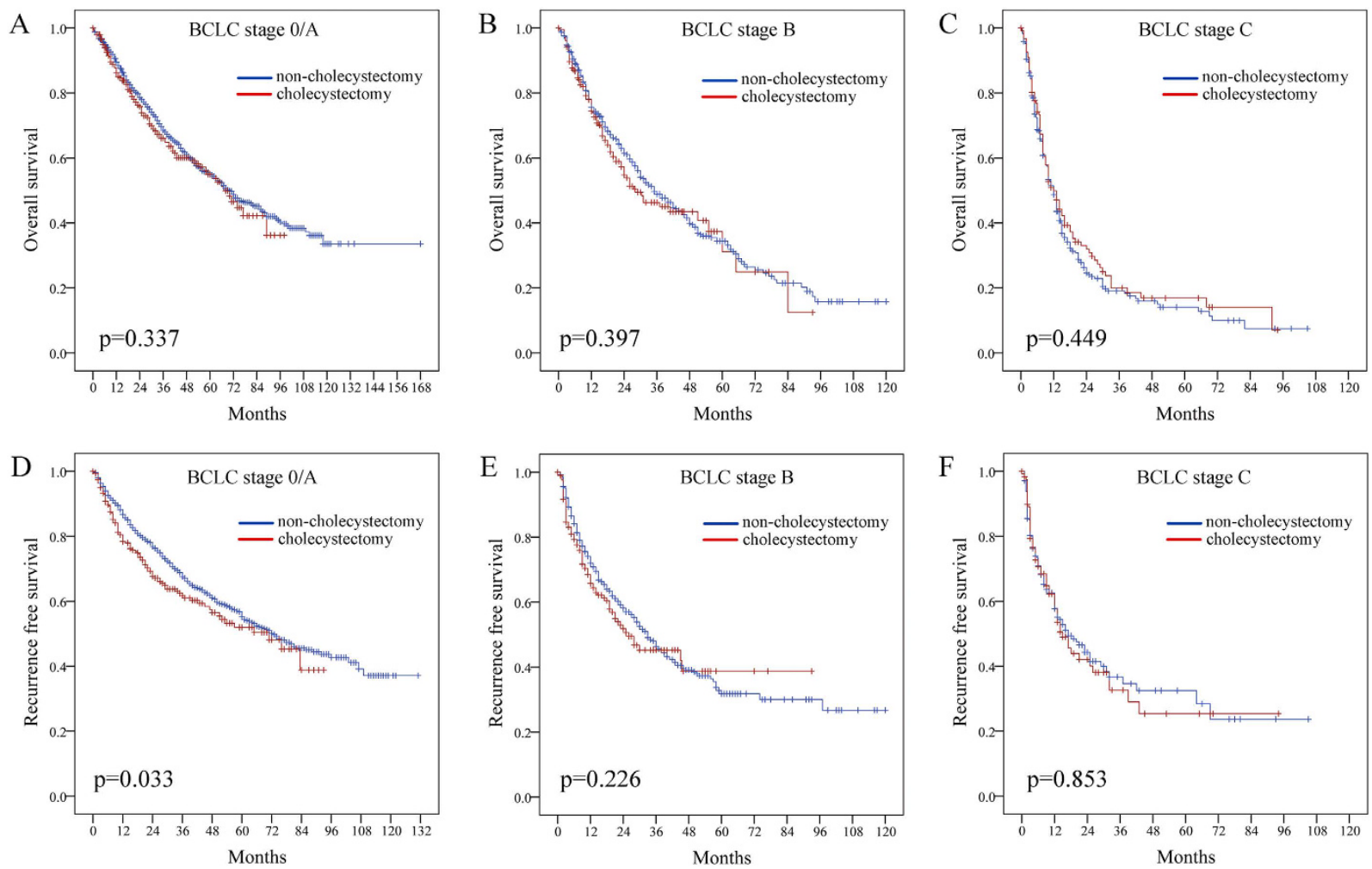

Figure 1. (A-C) Comparison of cumulative incidence of OS according to BCLC stage between cholecystectomy group and non-cholecystectomy group; (D-F) Comparison of cumulative incidence of RFS according to BCLC stage between cholecystectomy group and non-cholecystectomy group.

of $\operatorname{AFP}(792 / 1223$ vs 113/194, $\mathrm{p}=0.403)$, ALT (1771/244 vs 260/47, $\mathrm{p}=0.115)$ and GGT $(910 / 1105 \mathrm{vs} 124 / 183$, $\mathrm{p}=0.117)$. There were also no significant differences regarding the incidence of hepatitis $\mathrm{B}$ virus infection $(298 / 1717$ vs $47 / 260, p=0.811)$ and cirrhosis $(299 / 1716 v s 49 / 258, p=0.608)$ between these two groups for early stage patients.

Univariate and multivariate analysis of risk factors related to RFS of early stage HCC patients. Since for patients at early stage, the RFS rates of cholecystectomy group were significantly worse than those of non-cholecystectomy group, we further investigated whether cholecystectomy was an independent prognostic factor for RFS of patients at early stage. Table 3 demonstrated the prognostic factors related to postoperative RFS of early stage HCC patients in univariate and multivariate analysis. Multivariate analysis revealed that despite HBsAg ( $\mathrm{p}=0.031$, HR: 1.30, 95\% CI: 1.02-1.65), AFP level ( $<<0.001$, HR: 1.39, 95\% CI: 1.18-1.64), GGT level ( $\mathrm{p}=0.005$, HR: 1.25, 95\% CI: 1.07-1.47), tumor size ( $\mathrm{p}=0.002$, HR: $1.29,95 \%$ CI: $1.10-1.52)$, postoperative TACE ( $<$ < 0.001, HR: 3.39, 95\% CI: 2.89-3.99), cholecystectomy ( $\mathrm{p}=0.020$, HR: $1.29,95 \%$ CI: $1.04-1.59$ ) was also an independent prognostic factor for RFS of early stage HCC patients.

Univariate and multivariate analysis of predictive factors for early intrahepatic recurrence of early stage HCC patients. Univariate analysis of predictive factors for early and late intrahepatic recurrence of early stage HCC patients were listed in Table 4 and Table 5 respectively. Univariate analysis revealed that cholecystectomy was a risk factor for early recurrence ( $<<0.001$, HR: 1.69, 95\% CI: $1.27-2.24$ ), but had no predictive significance for late recurrence ( $\mathrm{p}=0.893$, HR: $1.03,95 \%$ CI: 0.68-1.55). Multivariate analysis (Table 4) revealed that besides AFP level ( $\mathrm{p}=0.001$, HR: 1.54, 95\% CI: 1.18-2.01), GGT level ( $\mathrm{p}=0.002$, HR: $1.49,95 \%$ CI: 1.16-1.92), tumor capsule ( $\mathrm{p}=0.016$, HR: $1.33,95 \%$ CI: 1.05-1.69) and tumor size ( $\mathrm{p}<0.001, \mathrm{HR}: 1.78,95 \%$ CI: 1.40-2.27), postoperative TACE ( $\mathrm{p}<0.001$, HR: $3.67,95 \%$ CI: $2.85-4.71)$ and IFN- $\alpha$ treatment $(\mathrm{p}=0.010$, HR: $1.89,95 \%$ CI: $1.17-3.05)$, cholecystectomy ( $\mathrm{p}=0.005$, HR: $1.52,95 \%$ CI: $1.13-2.03$ ) was also an independent risk factor for early recurrence of early stage HCC patients. In addition, for early stage patients that received minor resection, cholecystectomy still was an independent risk for early recurrence $(\mathrm{p}=0.048, \mathrm{HR}: 1.47,95 \%$ CI: 1.00-2.15, Supplementary Table 2). Only liver cirrhosis ( $p=0.001$, HR: 2.24, 95\% CI: 1.40-3.57, Table 5) and postoperative TACE ( $\mathrm{p}<0.001$, HR: $2.94,95 \%$ CI: $2.20-3.83$, Table 5) were independent risk factors for late recurrence of early stage HCC patients.

\section{Discussion}

HCC is a clear example of inflammation-related cancer as more than $90 \%$ of HCC arises in the context of hepatic injury and inflammation, particularly as the result of hepatitis B or C virus infection ${ }^{28-30}$. Chronic inflammation 


\begin{tabular}{|c|c|c|c|}
\hline \multirow[b]{2}{*}{ Variables (\%) } & \multicolumn{3}{|c|}{ HCC, $n=2322$} \\
\hline & $\begin{array}{l}\text { Non-cholecystectomy, } \\
\mathbf{n}=\mathbf{2 0 1 5}\end{array}$ & Cholecystectomy, $\mathbf{n}=\mathbf{3 0 7}$ & p value \\
\hline Gender & & & 0.220 \\
\hline female & $300(14.9)$ & $54(17.6)$ & \\
\hline male & $1715(85.1)$ & $253(82.4)$ & \\
\hline Age, yrs & & & 0.002 \\
\hline$\leq 50$ & $963(47.8)$ & $118(38.4)$ & \\
\hline$>50$ & $1052(52.2)$ & $189(61.6)$ & \\
\hline HBsAg & & & 0.811 \\
\hline negative & $298(14.8)$ & $47(15.3)$ & \\
\hline positive & $1717(85.2)$ & $260(84.7)$ & \\
\hline AFP, ng/mL & & & 0.403 \\
\hline$\leq 20$ & $792(39.3)$ & $113(36.8)$ & \\
\hline$>20$ & $1223(60.7)$ & $194(63.2)$ & \\
\hline AFP, mean, ng/mL & 1567.02 & 1889.07 & 0.166 \\
\hline$\leq 400$ & $1322(65.6)$ & $189(61.6)$ & \\
\hline$>400$ & $693(34.4)$ & $118(38.4)$ & \\
\hline ALT, U/L & & & 0.115 \\
\hline$\leq 75$ & $1771(87.9)$ & $260(84.7)$ & \\
\hline$>75$ & $244(12.1)$ & $47(15.3)$ & \\
\hline GGT, U/L & & & 0.117 \\
\hline$\leq 50$ & $910(45.2)$ & $124(40.4)$ & \\
\hline$>50$ & $1105(54.8)$ & $183(59.6)$ & \\
\hline Cirrhosis & & & 0.608 \\
\hline no & $299(14.8)$ & $49(16.0)$ & \\
\hline yes & $1716(85.2)$ & $258(84.0)$ & \\
\hline Child-Pugh Score & & & 0.157 \\
\hline A & $1882(93.4)$ & $280(91.2)$ & \\
\hline B & $133(6.6)$ & $27(8.8)$ & \\
\hline Tumor size, $\mathrm{cm}$ & & & $<0.001$ \\
\hline$\leq 5$ & $1317(65.4)$ & $160(52.1)$ & \\
\hline$>5$ & $698(34.6)$ & $147(47.9)$ & \\
\hline Tumor number & & & 0.260 \\
\hline single & $1952(96.9)$ & $301(98.0)$ & \\
\hline multiple & $63(3.1)$ & $6(2.0)$ & \\
\hline Tumor capsule & & & 0.091 \\
\hline no & $1263(62.7)$ & $177(57.7)$ & \\
\hline yes & $752(37.3)$ & $130(42.3)$ & \\
\hline Tumor differentiation & & & 0.663 \\
\hline I-II & $1455(72.2)$ & $218(71.0)$ & \\
\hline III-IV & $560(17.8)$ & $89(29.0)$ & \\
\hline
\end{tabular}

Table 2. Demographics and clinical characteristics of early stage (BCLC stage 0/A) HCC patients in cholecystectomy and non-cholecystectomy group.

following cholecystectomy is considered to associated with an increased risk of developing $\mathrm{HCC}^{2,3,5,31}$, but whether it will influence the outcome of HCC patients underwent curative resection remains to be clarified. In this study, stratified analysis of OS according to BCLC staging system demonstrated no significant difference between the cholecystectomy group and no-cholecystectomy group at early, intermediate or advanced stage, and only for patients at early stage, the RFS rates of cholecystectomy group were significantly worse than those of non-cholecystectomy group.

At present, the main cause for the dismal outcome of HCC is the high incidence of recurrence, and the overall prognosis depends largely on the pattern of recurrence ${ }^{32}$. The prognosis of early recurrence is much worse than that of late recurrence because early recurrence is primarily mediated by intrahepatic metastatic mechanism that is associated with tumor vascular invasion and by increased angiogenesis, whereas most of the late recurrences are multicentric occurrence in origin ${ }^{33,34}$. In this study, the early recurrence rate of cholecystectomy group was significantly higher than that of no-cholecystectomy group. However, when stratified by BCLC stage, the difference was significant only for patients at early stage, but not for patients at advanced stage.

Further multivariate analysis revealed that cholecystectomy was an independent risk factor for early recurrence but not for late recurrence of early stage HCC patients. It means that the impact of cholecystectomy on 


\begin{tabular}{|c|c|c|c|c|c|c|}
\hline \multirow[b]{2}{*}{ Variable } & \multicolumn{3}{|c|}{ Univariate analysis } & \multicolumn{3}{|c|}{ Multivariate analysis } \\
\hline & HR & $95 \% \mathrm{CI}$ & p value & HR & $95 \% \mathrm{CI}$ & p value \\
\hline \multicolumn{7}{|l|}{ Gender } \\
\hline female & 1 & & & 1 & & \\
\hline male & 1.20 & $0.96-1.50$ & 0.110 & 1.07 & $0.85-1.35$ & 0.565 \\
\hline \multicolumn{7}{|l|}{ Age years } \\
\hline$\leq 50$ & 1 & & & 1 & & \\
\hline$>50$ & 1.11 & $0.95-1.29$ & 0.183 & 1.15 & $0.98-1.34$ & 0.091 \\
\hline \multicolumn{7}{|l|}{ HBsAg } \\
\hline negative & 1 & & & 1 & & \\
\hline positive & 1.30 & $1.03-1.62$ & 0.024 & 1.30 & $1.02-1.65$ & 0.031 \\
\hline \multicolumn{7}{|l|}{ AFP, ng/mL } \\
\hline$\leq 20$ & 1 & & & 1 & & \\
\hline$>20$ & 1.45 & $1.24-1.70$ & $<0.001$ & 1.39 & $1.18-1.64$ & $<0.001$ \\
\hline \multicolumn{7}{|l|}{ ALT, U/L } \\
\hline$\leq 75$ & 1 & & & 1 & & \\
\hline$>75$ & 1.23 & $0.98-1.55$ & 0.068 & 1.04 & $0.83-1.31$ & 0.744 \\
\hline \multicolumn{7}{|c|}{ GGT, U/L (\%) } \\
\hline$\leq 50$ & 1 & & & 1 & & \\
\hline$>50$ & 1.41 & $1.21-1.64$ & $<0.001$ & 1.25 & $1.07-1.47$ & 0.005 \\
\hline \multicolumn{7}{|c|}{ Liver cirrhosis } \\
\hline no & 1 & & & 1 & & \\
\hline yes & 1.34 & $1.07-1.70$ & 0.013 & 1.24 & $0.97-1.57$ & 0.083 \\
\hline \multicolumn{7}{|c|}{ Child-Pugh Score } \\
\hline A & 1 & & & 1 & & \\
\hline $\mathrm{B}$ & 1.29 & $0.96-1.73$ & 0.094 & 1.17 & $0.86-1.57$ & 0.315 \\
\hline \multicolumn{7}{|c|}{ Tumor size, $\mathrm{cm}$} \\
\hline$\leq 5$ & 1 & & & 1 & & \\
\hline$>5$ & 1.42 & $1.22-1.65$ & $<0.001$ & 1.29 & $1.10-1.52$ & 0.002 \\
\hline \multicolumn{7}{|c|}{ Tumor number } \\
\hline single & 1 & & & 1 & & \\
\hline multiple & 1.02 & $0.65-1.62$ & 0.922 & 1.07 & $0.67-1.70$ & 0.777 \\
\hline \multicolumn{7}{|c|}{ Tumor capsule } \\
\hline yes & 1 & & & 1 & & \\
\hline no & 1.22 & $1.05-1.43$ & 0.011 & 1.13 & $0.96-1.32$ & 0.140 \\
\hline \multicolumn{7}{|c|}{ Tumor differentiation } \\
\hline I-II & 1 & & & 1 & & \\
\hline III-IV & 1.20 & $1.02--1.41$ & 0.032 & 1.08 & $0.91-1.27$ & 0.343 \\
\hline \multicolumn{7}{|c|}{ Vascular invasion } \\
\hline no & - & - & - & - & - & - \\
\hline yes & - & - & - & - & - & - \\
\hline \multicolumn{7}{|c|}{ Postoperative TACE (\%) } \\
\hline yes & 1 & & & 1 & & \\
\hline no & 1.38 & $1.34-1.43$ & $<0.001$ & 3.39 & $2.89-3.99$ & $<0.001$ \\
\hline \multicolumn{7}{|c|}{ IFN- $\alpha$ treatment } \\
\hline yes & 1 & & & 1 & & \\
\hline no & 1.11 & $0.86-1.42$ & 0.428 & 1.22 & $0.95-1.57$ & 0.121 \\
\hline \multicolumn{7}{|c|}{ Cholecystectomy } \\
\hline no & 1 & & & 1 & & \\
\hline yes & 1.25 & $1.02-1.54$ & 0.034 & 1.29 & $1.04-1.59$ & 0.020 \\
\hline
\end{tabular}

Table 3. Univariate and multivariate analysis of risk factors related to RFS of early stage (BCLC stage 0/A) HCC patients. HR: Hazard Ratio; CI: Confidence Interval; GGT, $\gamma$-glutamyl transferase; ALT, Alanine aminotransferase; AFP, a-fetoprotein.

postoperative recurrence diminished with increasing time after surgery. It's interesting that similar phenomenon was discovered in the investigations for risk of developing digestive cancers following cholecystectomy. It's reported that the rate ratios for developing digestive cancers were significantly high in the first year after cholecystectomy, but decreased rapidly over time and became non-significant as early as two years after 


\begin{tabular}{|c|c|c|c|c|c|c|}
\hline \multirow[b]{2}{*}{ Variable } & \multicolumn{3}{|c|}{ Univariate analysis } & \multicolumn{3}{|c|}{ Multivariate analysis } \\
\hline & HR & 95\% CI & p value & HR & 95\% CI & p value \\
\hline \multicolumn{7}{|l|}{ Gender } \\
\hline female & 1 & & & 1 & & \\
\hline male & 1.04 & $0.75-1.43$ & 0.835 & 1.05 & $0.75-1.46$ & 0.779 \\
\hline \multicolumn{7}{|l|}{ Age,years } \\
\hline$\leq 50$ & 1 & & & 1 & & \\
\hline$>50$ & 0.93 & $0.74-1.17$ & 0.517 & 1.03 & $0.81-1.31$ & 0.807 \\
\hline \multicolumn{7}{|l|}{ HBsAg } \\
\hline negative & 1 & & & 1 & & \\
\hline positive & 1.23 & $0.87-1.73$ & 0.243 & 1.32 & $0.92-1.91$ & 0.136 \\
\hline \multicolumn{7}{|c|}{ AFP, ng/mL } \\
\hline$\leq 20$ & 1 & & & 1 & & \\
\hline$>20$ & 1.80 & $1.39-2.32$ & $<0.001$ & 1.54 & $1.18-2.01$ & 0.001 \\
\hline \multicolumn{7}{|l|}{ ALT, U/L } \\
\hline$\leq 75$ & 1 & & & 1 & & \\
\hline$>75$ & 1.62 & $1.20-2.19$ & 0.002 & 1.25 & $0.92-1.70$ & 0.160 \\
\hline \multicolumn{7}{|c|}{ GGT, U/L (\%) } \\
\hline$\leq 50$ & 1 & & & 1 & & \\
\hline$>50$ & 1.80 & $1.41-2.30$ & $<0.001$ & 1.49 & $1.16-1.92$ & 0.002 \\
\hline \multicolumn{7}{|c|}{ Liver cirrhosis } \\
\hline no & 1 & & & 1 & & \\
\hline yes & 1.16 & $0.85-1.57$ & 0.356 & 1.10 & $0.80-1.52$ & 0.555 \\
\hline \multicolumn{7}{|c|}{ Child-Pugh Score } \\
\hline $\mathrm{A}$ & 1 & & & 1 & & \\
\hline $\mathrm{B}$ & 1.27 & $0.83-1.94$ & 0.278 & 1.28 & $0.83-1.97$ & 0.269 \\
\hline \multicolumn{7}{|c|}{ Tumor size, $\mathrm{cm}$} \\
\hline$\leq 5$ & 1 & & & 1 & & \\
\hline$>5$ & 2.26 & $1.80-2.85$ & $<0.001$ & 1.78 & $1.40-2.27$ & $<0.001$ \\
\hline \multicolumn{7}{|c|}{ Tumor number } \\
\hline single & 1 & & & 1 & & \\
\hline multiple & 1.75 & $0.72-4.24$ & 0.214 & 1.37 & $0.56-3.43$ & 0.493 \\
\hline \multicolumn{7}{|c|}{ Tumor capsule } \\
\hline yes & 1 & & & 1 & & \\
\hline no & 1.58 & $1.26-1.99$ & $<0.001$ & 1.33 & $1.05-1.69$ & 0.016 \\
\hline \multicolumn{7}{|c|}{ Tumor differentiation } \\
\hline I-II & 1 & & & 1 & & \\
\hline III-IV & 1.38 & $1.09-1.76$ & 0.009 & 1.19 & $0.93-1.53$ & 0.157 \\
\hline \multicolumn{7}{|c|}{ Vascular invasion } \\
\hline no & - & - & - & - & - & - \\
\hline yes & - & - & - & - & - & - \\
\hline \multicolumn{7}{|c|}{ Postoperative TACE (\%) } \\
\hline yes & 1 & & & 1 & & \\
\hline no & 3.77 & $2.94-4.84$ & 3.77 & 3.67 & $2.85-4.71$ & $<0.001$ \\
\hline \multicolumn{7}{|c|}{ IFN- $\alpha$ treatment } \\
\hline yes & 1 & & & 1 & & \\
\hline no & 1.83 & $1.14-2.95$ & 0.013 & 1.89 & $1.17-3.05$ & 0.010 \\
\hline \multicolumn{7}{|c|}{ Cholecystectomy } \\
\hline no & 1 & & & 1 & & \\
\hline yes & 1.69 & $1.27-2.24$ & $<0.001$ & 1.52 & $1.13-2.03$ & 0.005 \\
\hline
\end{tabular}

Table 4. Univariate and multivariate analysis of risk factors for early recurrence of early stage (BCLC stage 0/A) patients. HR, Hazard Ratio; CI, Confidence Interval; GGT, $\gamma$-glutamyl transferase; ALT, Alanine aminotransferase; AFP, a-fetoprotein.

cholecystectomy ${ }^{2,35-37}$. Since the time-scale of the association is too short, some researchers think that the association between cholecystectomy and subsequent digestive cancer is unlikely to be causal ${ }^{37}$.

However, an additional hypothesis is that cholecystectomy will lead to inflammation and accumulation of bile, both of which can act as carcinogens to promote cancer recurrence. Studies demonstrate that removal of the gallbladder will leads to Sphincter of Oddi (SO) dysfunction, which in turn will cause dilation of the CBD, elevated 


\begin{tabular}{|c|c|c|c|c|c|c|}
\hline \multirow[b]{2}{*}{ Variable } & \multicolumn{3}{|c|}{ Univariate analysis } & \multicolumn{3}{|c|}{ Multivariate analysis } \\
\hline & HR & $95 \% \mathrm{CI}$ & $p$ value & HR & $95 \% \mathrm{CI}$ & p value \\
\hline \multicolumn{7}{|l|}{ Gender } \\
\hline female & 1 & & & 1 & & \\
\hline male & 1.65 & $1.08-2.51$ & 0.021 & 1.41 & $0.92-2.17$ & 0.119 \\
\hline \multicolumn{7}{|l|}{ Age,years } \\
\hline$\leq 50$ & 1 & & & 1 & & \\
\hline$>50$ & 1.28 & $0.99-1.66$ & 0.057 & 1.29 & $0.98-1.68$ & 0.066 \\
\hline \multicolumn{7}{|l|}{ HBsAg } \\
\hline negative & 1 & & & 1 & & \\
\hline positive & 1.16 & $0.82-1.64$ & 0.399 & 1.16 & $0.81-1.67$ & 0.420 \\
\hline \multicolumn{7}{|l|}{ AFP, ng/mL } \\
\hline$\leq 20$ & 1 & & & 1 & & \\
\hline$>20$ & 1.09 & $0.84-1.41$ & 0.514 & 1.13 & $0.86-1.47$ & 0.380 \\
\hline \multicolumn{7}{|l|}{ ALT, U/L } \\
\hline$\leq 75$ & 1 & & & 1 & & \\
\hline$>75$ & 1.23 & $0.77-1.97$ & 0.388 & 1.49 & $0.92-2.39$ & 0.105 \\
\hline \multicolumn{7}{|c|}{ GGT, U/L (\%) } \\
\hline$\leq 50$ & 1 & & & 1 & & \\
\hline$>50$ & 1.28 & $0.99-1.65$ & 0.058 & 1.17 & $0.90-1.52$ & 0.255 \\
\hline \multicolumn{7}{|c|}{ Liver cirrhosis } \\
\hline no & 1 & & & 1 & & \\
\hline yes & 2.16 & $1.38-3.38$ & 0.001 & 2.24 & $1.40-3.57$ & 0.001 \\
\hline \multicolumn{7}{|c|}{ Child-Pugh Score } \\
\hline A & 1 & & & 1 & & \\
\hline $\mathrm{B}$ & 1.34 & $0.78-2.31$ & 0.283 & 1.40 & $0.81-2.44$ & 0.230 \\
\hline \multicolumn{7}{|c|}{ Tumor size, $\mathrm{cm}$} \\
\hline$\leq 5$ & 1 & & & 1 & & \\
\hline$>5$ & 1.04 & $0.79-1.38$ & 0.766 & 1.07 & $0.80-1.42$ & 0.659 \\
\hline \multicolumn{7}{|c|}{ Tumor number } \\
\hline single & 1 & & & 1 & & \\
\hline multiple & 1.57 & $0.83-2.96$ & 0.164 & 1.61 & $0.84-3.07$ & 0.153 \\
\hline \multicolumn{7}{|c|}{ Tumor capsule } \\
\hline yes & 1 & & & 1 & & \\
\hline no & 1.10 & $0.83-1.45$ & 0.525 & 1.13 & $0.85-1.50$ & 0.403 \\
\hline \multicolumn{7}{|c|}{ Tumor differentiation } \\
\hline I-II & 1 & & & 1 & & \\
\hline III-IV & 1.15 & $0.88-1.51$ & 0.317 & 1.04 & $0.79-1.38$ & 0.780 \\
\hline \multicolumn{7}{|c|}{ Vascular invasion } \\
\hline no & - & - & - & - & - & - \\
\hline yes & - & - & - & - & - & - \\
\hline \multicolumn{7}{|c|}{ Postoperative TACE (\%) } \\
\hline yes & 1 & & & 1 & & \\
\hline no & 2.91 & $2.25-3.77$ & $<0.001$ & 2.94 & $2.20-3.83$ & $<0.001$ \\
\hline \multicolumn{7}{|c|}{ IFN- $\alpha$ treatment } \\
\hline yes & 1 & & & 1 & & \\
\hline no & 1.37 & $0.97-1.94$ & 0.074 & 1.24 & $0.87-1.77$ & 0.229 \\
\hline \multicolumn{7}{|c|}{ Cholecystectomy } \\
\hline no & 1 & & & 1 & & \\
\hline yes & 1.19 & $0.79-1.77$ & 0.404 & 1.01 & $0.67-1.53$ & 0.959 \\
\hline
\end{tabular}

Table 5. Univariate and multivariate analysis of risk factors for late recurrence of early stage (BCLC stage 0/A) patients. HR, Hazard Ratio; CI, Confidence Interval; GGT, $\gamma$-glutamyl transferase; ALT, Alanine aminotransferase; AFP, a-fetoprotein.

bile duct pressure, and subsequent cholestasis and chronic inflammation ${ }^{6-11}$. Inflammation-mediated tumor angiogenesis has been confirmed by various studies and release of inflammation related cytokines, chemokines, growth factors plays decisive roles in cancer invasion, recurrence and metastasis ${ }^{5,16-18}$. In addition, the accumulation of bile and secondary bile acids, in particular, deoxycholic acid, can act as carcinogens and promote the 
recurrence and occurrence of cancers ${ }^{38,39}$. However, because of internal compensation, SO could adjust to the changes produced by cholecystectomy and SO motility will recover in several months after cholecystectomy to avoid subsequent cholestasis, CBD hypertension and inflammation ${ }^{40,41}$. This may not only explains why most patients with postcholecystectomy syndrome recover in a year after operation, but explains the short-term association of cholecystectomy and increased risk of tumorigenesis or recurrence.

There seems to be a consensus among some surgeons that patients with HCC should simultaneously undergo cholecystectomy at the time of resection for HCC, not only because performing a subsequent cholecystectomy on a patient who has undergone prior hepatic surgery is technically difficult ${ }^{42}$, but also for fear of gangrenous cholecystitis after transcatheter arterial chemoembolization (TACE) treatment. Though inadvertent reflux of the embolic material into the cystic artery after TACE has been reported ${ }^{43}$, super selective embolization technique has significantly reduce the risk of cholecystitis and the reported incidence is lower than $5 \%{ }^{44}$. In most cases, management can be conservative, and cholecystectomy is not necessary ${ }^{44}$.

In addition, the most common extrahepatic metastatic sites of HCC are lung, abdominal lymph nodes, bone and adrenal gland, direct invasion or metastasis to the gallbladder is rare ${ }^{42,45}$. It has been suggested that gallbladder cancer can easily invade the liver because there is no peritoneum between the gallbladder and the liver fossa. On the other hand, HCC does not normally invade the gallbladder because HCC rarely destroys the muscle layer or the collagen fibers of the gallbladder wall ${ }^{42}$. In accordance with the previous study ${ }^{42}$, the prevalence of invasion or metastasis from HCC to the gallbladder was only $0.2 \%$ (9/3933) in our study, such low incidence dose does not warrant routine cholecystectomy during resection for HCC unless gallbladder metastasis is suspected.

To the best of our knowledge, no previous study has addressed a possible relation between cholecystectomy and increased risk of early recurrence of early stage HCC. Since cholecystectomy is associated with early recurrence and poorer RFS of early stage HCC patients that underwent curative resection, routine removal of normal gallbladder during HCC resection may be avoided for patients at early stage, and the indication of cholecystectomy may be restricted to patients with gallbladder diseases or metastasis. However, because of the retrospective nature of this study, our findings need to be confirmed by future prospective research before conclusions can be drawn. Though it is too early to consider any potential clinical recommendations, our findings would question the indication of routine cholecystectomy during HCC resection.

Patients and methods. Study population. This study was approved by the Ethic Committee of Zhongshan Hospital and Qilu Hospital, performed in accordance with the approved guidelines, and informed consent was obtained from all subjects.

Data of HCC patients treated by curative resection for HCC in liver cancer institute of Fudan University and Qilu Hospital of Shandong University between January 1999 and December 2009 were retrieved from a prospectively collected database and were retrospectively analyzed. Curative resection was defined as complete resection of all tumor nodules and the cut surface being free of cancer by histologic examination; having no cancerous thrombus in the portal vein, hepatic veins, or bile duct; and having no extrahepatic metastasis ${ }^{46,47}$. All pathologic specimens were reviewed by 2 pathologists to confirm the diagnosis of HCC. The histologic grade of tumor differentiation was assigned by the Edmondson grading system. The staging of tumors was determined according to the BCLC staging system. Liver function was assessed by Child-Pugh score system. All the HBV-related HCC patients did not receive any anti-HBV treatment except IFN-a treatment.

Factors that may potentially related to survival and recurrence were selected in this study on the basis of previous studies, including age ( $\leq 50$ or $>50$ years), gender (male or female), cirrhosis (yes or no), Child-Pugh Score (A or B), tumor size $(\leq 5$ or $>5 \mathrm{~cm})$, number of tumor nodules (single or multiple), tumor capsule (yes or no), vascular invasion (yes or no), tumor differentiation (Edmondson's classification I/II or III/IV), TACE or IFN- $\alpha$ treatment (yes or no). Preoperative laboratory values of serum alanine aminotransferase (ALT) ( $\leq 75$ or $>75 \mathrm{U} / \mathrm{L})$, $\gamma$-glutamyl transpeptidase (GGT) $(\leq 50$ or $>50 \mathrm{U} / \mathrm{L})$, and a-fetoprotein (AFP) concentration $(\leq 20 \mathrm{or}>20 \mathrm{ng} / \mathrm{mL})$ were also included in analysis, using the upper limit of the normal values in our hospital as the cutoff values. All tumor related factors were determined by pathological examination of resected tissue.

Follow-up. Patients were followed up every 2 months after operation in outpatient clinics and monitored prospectively for recurrence by a standard protocol that included serum AFP, liver function, and ultrasonography or contrastenhanced computed tomography (CT). CT scan of the abdomen was performed every 6 months. Bone scan or magnetic resonance imaging (MRI) was performed if localized bone pain was reported. Intrahepatic recurrence was defined as a new lesion in the remnant liver with typical imaging appearance in CT/MRI and an elevated AFP level according to EASL proposed criteria for HCC. Ultrasound-guided fine-needle biopsy is sometimes needed to confirm the diagnosis when imaging is atypical. Recurrent cases were divided into early or late recurrent group, using 1 year as the cutoff value, as suggested by Poon's study ${ }^{34}$. In this study, only the first recurrence was included.

Statistical analysis. The $\chi^{2}$ test or Fisher's exact probability was used for categorical variables and Student's t test was used for continuous variables. Cumulative overall survival rate was calculated by the Kaplan-Meier method and compared by the log-rank test. OS was calculated from the date of resection to the date of death regardless of reason of death. RFS was calculated from the date of resection to the date when tumor recurrence was diagnosed, or from date of the resection to the last visit, if recurrence was not diagnosed, the cases were censored at the date of death or the last date of follow-up. The Cox proportional hazards model was used to determine the independent factors on survival and recurrence. Statistical analyses were performed by SPSS 19.0 (SPSS Inc., Chicago, IL). Two-tailed $\mathrm{P}<0.05$ was considered statistically significant. 


\section{References}

1. Wood, R., Fraser, L. A., Brewster, D. H. \& Garden, O. J. Epidemiology of gallbladder cancer and trends in cholecystectomy rates in Scotland, 1968-1998. Eur J Cancer 39, 2080-2086 (2003).

2. Nogueira, L. et al. Gallstones, cholecystectomy, and risk of digestive system cancers. Am J Epidemiol 179, 731-739 (2014).

3. Lagergren, J., Mattsson, F., El-Serag, H. \& Nordenstedt, H. Increased risk of hepatocellular carcinoma after cholecystectomy. Br J Cancer 105, 154-156 (2011).

4. Guo, L. et al. Cholelithiasis, cholecystectomy and risk of hepatocellular carcinoma: a meta-analysis. J Cancer Res Ther 10, 834-838 (2014).

5. Liu, Y. et al. Risk of primary liver cancer associated with gallstones and cholecystectomy: a meta-analysis. PLoS One 9, e109733 (2014).

6. Csendes, A. et al. Pressure measurements in the biliary and pancreatic duct systems in controls and in patients with gallstones, previous cholecystectomy, or common bile duct stones. Gastroenterology 77, 1203-1210 (1979).

7. Tanaka, M., Ikeda, S. \& Nakayama, F. Change in bile duct pressure responses after cholecystectomy: loss of gallbladder as a pressure reservoir. Gastroenterology 87, 1154-1159 (1984).

8. Sarkisian, S. A. \& Mcgowan, J. M. The relation of bacteria in the bile to biliary dynamics, cholangitis, and postcholecystectomy syndrome. Surgery 35, 565-573 (1954).

9. Madureira, F. A., Manso, J. E., Madureira, F. D. \& Iglesias, A. C. Inflammation in laparoendoscopic single-site surgery versus laparoscopic cholecystectomy. Surg Innov 21, 263-268 (2014).

10. Sista, F. et al. Ultrasonic versus standard electric dissection in laparoscopic cholecystectomy in patients with acute calculous cholecystitis, complicated by peritonitis: influence on the postoperative systemic inflammation and immune response. A prospective randomized study. J Laparoendosc Adv Surg Tech A 24, 151-158 (2014).

11. Li, Y., He, R., Chen, S. \& Qu, Y. Effect of dexmedetomidine on early postoperative cognitive dysfunction and peri-operative inflammation in elderly patients undergoing laparoscopic cholecystectomy. Exp Ther Med 10, 1635-1642 (2015).

12. Barashi, N. et al. Inflammation-induced hepatocellular carcinoma is dependent on CCR5 in mice. Hepatology 58, 1021-1030 (2013).

13. Tarao, K. et al. Role of increased DNA synthesis activity of hepatocytes in multicentric hepatocarcinogenesis in residual liver of hepatectomized cirrhotic patients with hepatocellular carcinoma. Jpn J Cancer Res 85, 1040-1044 (1994).

14. Li, T. et al. Risk factors, prognosis, and management of early and late intrahepatic recurrence after resection of primary clear cell carcinoma of the liver. Ann Surg Oncol 18, 1955-1963 (2011).

15. El-Serag, H. B. \& Rudolph, K. L. Hepatocellular carcinoma: epidemiology and molecular carcinogenesis. Gastroenterology 132, 2557-2576 (2007).

16. Wang, Q. et al. The Severity of Liver Fibrosis Influences the Prognostic Value of Inflammation-Based Scores in Hepatitis B-Associated Hepatocellular Carcinoma. Ann Surg Oncol 22, 1125-1132 (2015).

17. Poon, R. T. et al. Tumor microvessel density as a predictor of recurrence after resection of hepatocellular carcinoma: a prospective study. J Clin Oncol 20, 1775-1785(2002).

18. Aravalli, R. N. \& Greten, T. F. FoxC1: Novel Regulator of Inflammation-Induced Metastasis in Hepatocellular Carcinoma. Gastroenterology 149, 861-863 (2015).

19. Li, T. et al. Clinical characteristics, outcome, and risk factors for early and late intrahepatic recurrence of female patients after curative resection of hepatocellular carcinoma. Surgery 156, 651-660 (2014).

20. Zhou, D. et al. A monocyte/granulocyte to lymphocyte ratio predicts survival in patients with hepatocellular carcinoma. Sci Rep 5, 15263 (2015)

21. Li, T. et al. Hepatitis B virus surface antigen-negative and hepatitis C virus antibody-negative hepatocellular carcinoma: clinical characteristics, outcome, and risk factors for early and late intrahepatic recurrence after resection. Cancer 119, 126-135 (2013).

22. $\mathrm{Li}$, T. et al. Positive $\mathrm{HBcAb}$ is associated with higher risk of early recurrence and poorer survival after curative resection of $\mathrm{HBV}$ related HCC. Liver Int 36, 284-292 (2016).

23. Sohn, W. et al. HBV DNA and HBsAg levels as risk predictors of early and late recurrence after curative resection of HBV-related hepatocellular carcinoma. Ann Surg Oncol 21, 2429-2435 (2014).

24. Huang, G. et al. Prediction of hepatocellular carcinoma recurrence in patients with low hepatitis B virus DNA levels and high preoperative hepatitis B surface antigen levels. JAMA Surg 149, 519-527 (2014).

25. Chan, S. L. et al. Use of antiviral therapy in surveillance: impact on outcome of hepatitis B-related hepatocellular carcinoma. Liver Int 32, 271-278 (2012).

26. Huang, G. et al. Antiviral therapy improves postoperative survival in patients with hepatocellular carcinoma: a randomized controlled trial. Ann Surg 261, 56-66 (2015).

27. Yeh, C. C. et al. Nonsteroidal anti-inflammatory drugs are associated with reduced risk of early hepatocellular carcinoma recurrence after curative liver resection: a nationwide cohort study. Ann Surg 261, 521-526 (2015).

28. Colombo, M. \& Sangiovanni, A. Treatment of hepatocellular carcinoma: beyond international guidelines. Liver Int 35, 129-138 (2015).

29. Yu, L. et al. Quantitative assessment of common genetic variations in HLA-DP with hepatitis B virus infection, clearance and hepatocellular carcinoma development. Sci Rep 5, 14933 (2015).

30. Triolo, M., Della, C. C. \& Colombo, M. Impact of HBV therapy on the incidence of hepatocellular carcinoma. Liver Int 34, 139-145 (2014).

31. Kao, W. Y. et al. Risk of hepato-biliary cancer after cholecystectomy: a nationwide cohort study. J Gastrointest Surg 17, 345-351 (2013).

32. Naito, S. et al. Postoperative recurrence pattern and prognosis of patients with hepatocellular carcinoma, with particular reference to the hepatitis viral infection status. Liver Int 34, 802-813 (2014).

33. Poon, R. T. et al. Different risk factors and prognosis for early and late intrahepatic recurrence after resection of hepatocellular carcinoma. Cancer 89, 500-507 (2000).

34. Poon, R. T. et al. Intrahepatic recurrence after curative resection of hepatocellular carcinoma: long-term results of treatment and prognostic factors. Ann Surg 229, 216-222 (1999).

35. Goldacre, M. J. et al. Cancer after cholecystectomy: record-linkage cohort study. Br J Cancer 92, 1307-1309 (2005).

36. Nordenstedt, H. et al. Gallstones and cholecystectomy in relation to risk of intra- and extrahepatic cholangiocarcinoma. Br J Cancer 106, 1011-1015 (2012).

37. Goldacre, M. J. et al. Association between cholecystectomy and intestinal cancer: a national record linkage study. Ann Surg 256, 1068-1072 (2012).

38. Perez, M. J. \& Briz, O. Bile-acid-induced cell injury and protection. World J Gastroenterol 15, 1677-1689 (2009).

39. Zhang, W. et al. A weighted relative difference accumulation algorithm for dynamic metabolomics data: long-term elevated bile acids are risk factors for hepatocellular carcinoma. Sci Rep 5, 8984 (2015).

40. Li, F. et al. Effects of cholecystectomy on the changes of motility of Beagle dogs' sphincter of Oddi. Acta Cir Bras 29, 237-244 (2014).

41. Fan, M. M., Li, F. \& Zhang, X. W. Changes of the sphincter of Oddi motility in dog after cholecystectomy. J Dig Dis 13, 40-46 (2012).

42. Lai, S. W. Pathologic evaluation of gallbladder in patients who underwent cholecystectomy and hepatic resection for hepatocellular carcinoma. Am J Med Sci 341, 305-307 (2011). 
43. Karavias, D. et al. Gangrenous Cholecystitis Related to Transcatheter Arterial Chemoembolization (TACE) Treatment for Hepatocellular Carcinoma. J Gastrointest Surg 19, 2093-2095 (2015).

44. Wagnetz, U. et al. Acute ischemic cholecystitis after transarterial chemoembolization of hepatocellular carcinoma: incidence and clinical outcome. J Comput Assist Tomogr 34, 348-353 (2010).

45. Choi, S. H. et al. FDG-PET predicts outcomes of treated bone metastasis following palliative radiotherapy in patients with hepatocellular carcinoma. Liver Int 34, 1118-1125 (2014).

46. Li, T. et al. Staging, prognostic factors and adjuvant therapy of intrahepatic cholangiocarcinoma after curative resection. Liver Int 34, 953-960 (2014).

47. Wang, C. H. et al. TMPRSS4 facilitates epithelial-mesenchymal transition of hepatocellular carcinoma and is a predictive marker for poor prognosis of patients after curative resection. Sci Rep 5, 12366 (2015).

\section{Acknowledgements}

This work was supported by the grants from the National Natural Science Foundation of China (Grant No. 81572328) and Major Program of Shandong Provincial Natural Science Foundation (Grant No. ZR2014HZ002).

\section{Author Contributions}

T.L. and X.-T.Z. designed the study, analyzed the results and drafted the manuscript. S.-K.W., J.Z., Z.-R.D., H.-C.S., J.F. and Q.-H.Y. helped to data collection and analysis. Z.-R.D. and Z.-L.Z. kindly offered valuable suggestions about the study design. All authors reviewed the manuscript.

\section{Additional Information}

Supplementary information accompanies this paper at http://www.nature.com/srep

Competing financial interests: The authors declare no competing financial interests.

How to cite this article: $\mathrm{Li}$, T. et al. Cholecystectomy is associated with higher risk of early recurrence and poorer survival after curative resection for early stage hepatocellular carcinoma. Sci. Rep. 6, 28229; doi: 10.1038/ srep28229 (2016).

(c) (i) This work is licensed under a Creative Commons Attribution 4.0 International License. The images or other third party material in this article are included in the article's Creative Commons license, unless indicated otherwise in the credit line; if the material is not included under the Creative Commons license, users will need to obtain permission from the license holder to reproduce the material. To view a copy of this license, visit http://creativecommons.org/licenses/by/4.0/ 